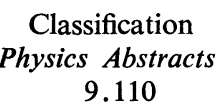

\title{
RÉALISATION D'UN APPAREILLAGE D'ÉLECTROLYSE EN PHASE SOLIDE. APPLICATION A LA CRISTALLOGENĖSE DES TERRES RARES ET DU TITANE
}

\author{
R. ALÉONARD, J. L. FÉRON et P. MORIN \\ Laboratoire de Magnétisme, CNRS \\ BP 166, 38042 Grenoble Cedex, France
}

(Reçu le 21 mai 1973)

\begin{abstract}
Résumé. - Nous décrivons un appareillage d'électrotransport en phase solide, réalisé dans un but de purification et de cristallogenèse. Cette méthode est bien adaptée aux métaux très réactifs car les contacts chauds entre l'échantillon et l'appareillage sont limités. Nous avons appliqué cette technique à la cristallogenèse des terres rares, métalliques ou en solution solide dans l'yttrium, ainsi qu'à celle du titane.

Abstract. - An electrotransport apparatus capable of sample purification as well as of crystal growth is described. This method is well suited for metals of high reactivity because of the limited hot contacts. This technique is applied to the crystal growth of titanium, rare earths and also of yttrium-rare earth solid solutions.
\end{abstract}

1. Introduction. - Le mouvement d'atomes d'impuretés dans un métal à l'état solide soumis à un champ électrique, est un phénomène connu depuis de nombreuses années. La première observation de l'électrotransport est attribuée à Gérardin qui, en 1861, réalisa l'électrolyse d'un alliage étain-plomb. Une revue précise des aspects tant théoriques qu'expérimentaux a été faite par Verhoeven [1]. Les atomes d'impuretés en solution entraînent les joints de grain dans leur migration; outre la purification, il en résulte une recristallisation primaire. Ce procédé a été mis en œuvre pour le zirconium [2], l'yttrium [3], [4], les métaux de terres rares [5], [6], [7] et certains métaux réactifs [8], [9]. Pour notre part nous l'avons appliqué aux terres rares, métalliques ou en solution solide, et au titane.

2. Méthode de purification. - Les matériaux polycristallins de départ sont préparés par une méthode de pseudo-lévitation [10]; l'échantillon, chauffé par haute fréquence, est fondu puis brassé électrodynamiquement sans contact avec un creuset; il est ensuite coulé dans une lingotière en cuivre refroidie. Cette méthode évite toute contamination et assure une bonne homogénéité des alliages.

Nous avons représenté sur la figure 1 une coupe du four à électrolyse en phase solide. Une alimentation 600 ampères-4 volts, stabilisée à mieux de $10^{-4}$ fournit le courant nécessaire. Une électrode en cuivre, refroidie et coulissant sur des paliers en téflon, permet de

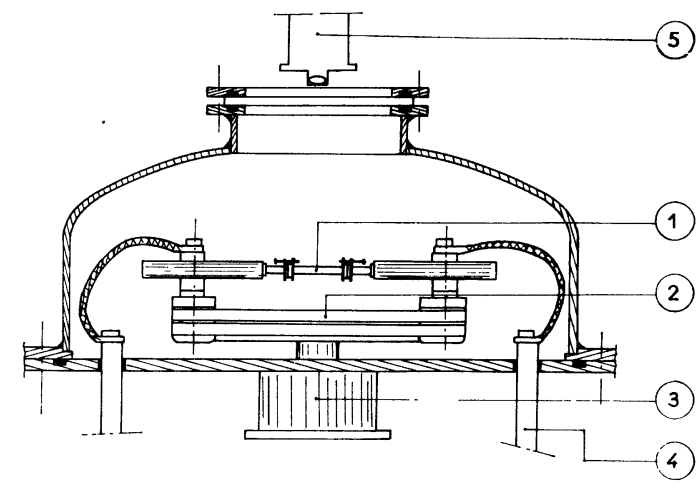

Fig. 1. - Schéma de l'appareil d'électrolyse en phase solide :

1. Echantillon.

2. Banc d'optique.

3. Pompage secondaire.

4. Traversées électriques.

5. Lunette pyrométrique.

compenser les dilatations de l'échantillon; du mercure assure les contacts thermiques et électriques (Fig. 2). Une pièce en molybdène sert de liaison entre l'électrode en cuivre et l'échantillon et détermine le gradient thermique.

Nous mesurons les températures avec un pyromètre optique à radiation totale. Son étalonnage est obtenu par la variation de la résistivité de l'échantillon étudiée aux transformations de phase, afin de tenir compte des modifications du pouvoir émissif de l'état de surface. Le pyromètre, couplé à l'alimentation stabi- 


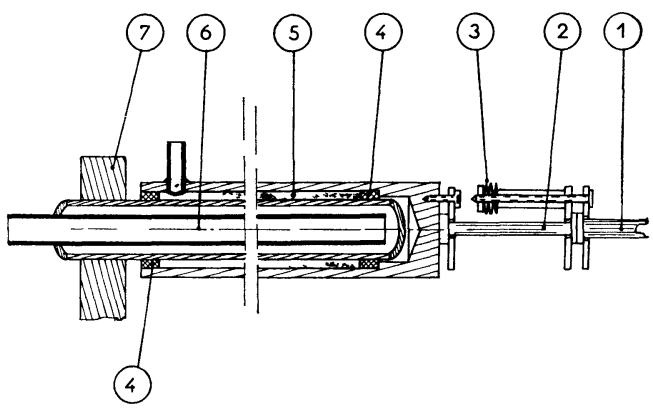

Fig. 2. - Détail de l'électrode coulissante :

1. Echantillon.

2. Electrode en molybdène.

3 . Rondelles ressorts de serrage.

4. Paliers en téflon.

5. Mercure.

6. Circulation d'eau.

7. Amenée de courant.

lisée, permet d'effectuer des paliers ou des descentes programmées de la température.

Les recuits se font soit sous vide, soit sous atmosphère de haute pureté.

\section{TABLEAU I}

Analyse d'yttrium monocristallin après électrolyse à $1350^{\circ} \mathrm{C}$ pendant $100 \mathrm{~h}$

\begin{tabular}{|c|c|c|c|}
\hline \multirow{2}{*}{ Eléments } & \multicolumn{3}{|c|}{ Analyse en p.p.m. } \\
\hline & $\begin{array}{l}1 \mathrm{~cm} \text { de la } \\
\text { cathode }\end{array}$ & $\begin{array}{l}2 \mathrm{~cm} \text { de la } \\
\text { cathode }\end{array}$ & $\begin{array}{l}3 \mathrm{~cm} \mathrm{de} \mathrm{la} \\
\text { cathode }\end{array}$ \\
\hline oxygène & 800 & 1500 & 3000 \\
\hline azote & 90 & 130 & 350 \\
\hline fluor & 150 & 130 & 950 \\
\hline fer & 3 & 10 & 260 \\
\hline nickel & 1,6 & 6 & 27 \\
\hline cobalt & 2,5 & 3 & 5 \\
\hline cuivre & 60 & 95 & 130 \\
\hline sodium & 90 & 7 & 8 \\
\hline magnesium & 70 & 25 & 10 \\
\hline lanthane & 8 & 9 & 8 \\
\hline gadolinium & 6 & 10 & 13 \\
\hline terbium & 0,6 & 0,6 & $0,3 !$ \\
\hline holmium & 0,2 & 0,3 & 0,2 \\
\hline erbium & 0,6 & 0,8 & 0,8 \\
\hline thulium & 10 & 15 & 5,5 \\
\hline
\end{tabular}

3. Application à l'yttrium et aux métaux de terres rares. - L'échantillon cylindrique ( $8 \mathrm{~mm}$ de diamètre) d'yttrium polycristallin $(99,5 \%)$ est maintenu pendant une centaine d'heures à une température de $1350^{\circ} \mathrm{C}$; la densité de courant est de $10 \mathrm{~A} / \mathrm{mm}^{2}$. Après électrolyse l'échantillon est très brillant, seule l'anode est grisâtre et cassante du fait de la présence de $\mathrm{Y}_{2} \mathrm{O}_{3}$. Il est formé d'un grain monocristallin de $2,5 \mathrm{~cm}$ de long sur toute la section; les taches de réflexion sur les clichés de Laüe sont particulièrement fines.

A titre indicatif, les résultats d'une analyse par spectrométrie de masse à étincelles à 1,2 et $3 \mathrm{~cm}$ de la cathode sont portés dans le tableau I. Les atomes d'impuretés dans la matrice d'yttrium se polarisent sous l'action du champ électrique : les métaux plus électronégatifs que l'yttrium $(\mathrm{Fe}, \mathrm{Ni}, \mathrm{Co}, \mathrm{Cu})$ se dirigent vers l'anode alors que les métaux moins électronégatifs $(\mathrm{Na}, \mathrm{Mg})$ vont vers la cathode. Nous ne notons aucun déplacement des métaux de terres rares dont l'électronégativité est identique à celle de l'yttrium. Le mouvement de l'oxygène vers l'anode est particulièrement remarquable.

De la même façon nous avons obtenu, par un recuit de $1300^{\circ} \mathrm{C}$ pendant $100 \mathrm{~h}$, un cristal de gadolinium de $20 \mathrm{~mm}$ de long et de $6 \mathrm{~mm}$ de diamètre. Le fait que les terres rares ne migrent pas dans l'yttrium, nous a également permis de préparer des cristaux de solution solide à faibles concentrations de terres rares, du type $\mathrm{Y}_{1-\mathrm{x}} \mathrm{Dy}_{\mathrm{x}}$.

4. Application au titane. - La grande réactivité du titane à haute température et l'existence d'une transformation $\mathrm{BCC} \rightarrow \mathrm{HCP}$ à $880^{\circ} \mathrm{C}$, rendent difficile l'obtention de monocristaux de titane par des méthodes traditionnelles. L'électrolyse en phase solide où les contacts chauds de l'échantillon avec l'appareillage sont très limités [11] nous a paru une méthode bien adaptée. $\mathrm{La}$ recristallisation primaire s'effectue dans la phase BCC, par un recuit d'une dizaine $\mathrm{d}$ 'heures à $1500^{\circ} \mathrm{C}$. Une descente lente de $3 \% / \mathrm{h}$ permet le passage de la transformation de phase sans recristallisation secondaire. Les grains monocristallins de la phase hexagonale, d'environ $2 \mathrm{~cm}$ de long et $8 \mathrm{~mm}$ de diamètre ne présentent pas d'imperfection aux rayons $\mathrm{X}$.

5. Conclusion. - L'électrolyse en phase solide, qui allie champ électrique et haute température, est donc un procédé efficace de purification et de recristallisation primaire. Les cristaux obtenus sont de très bonne qualité ; leur taille, dans notre cas, n'est limitée que par la section du barreau. La mise en œuvre de l'appareillage est simple. L'absence à haute température de contacts importants entre échantillon et appareillage, rend cette méthode particulièrement adaptée à la cristallogenèse des métaux réactifs. Outre les métaux étudiés, elle doit conduire à des résultats satisfaisants pour les métaux des groupes III B, IV B et V B de la table périodique. 


\section{Bibliographie}

[1] Verhoeven, J. D., Met. Rev. 8 (1963) 311.

[2] De Boer, J. H., FAST, J. D., Rec. Trav. Chim. 59 (1940) 161.

[3] Williams, J. M., Huffine, C. L., Nucl. Sci. Eng. 9 (1961) 500.

[4] Carlson, O. N., Schmidt, F. A., Peterson, D. T., J. Less Common Metals 10 (1966) 1.

[5] Marchant, J. D., Shedd, E. S., Henrie, T. A., Report of investigations 6894, Bureau of Mines (USA).

[6] Peterson, D. T., Schmidt, F. A., J. Less Common Metals 18 (1969) 111.
[7] Peterson, D. T., Schmidt, F. A., J. Less Common Metals 29 (1972) 321.

[8] Schmidt, F. A., Warner, J. C., J. Less Common Metals 13 (1967) 493.

[9] Schmidt, F. A., Carlson, O. N., J. Less Common Metals 26 (1972) 247.

[10] Bethoux, O., Cornut, B., Ferrari, M., Revue Phys. Appl. 5 (1970) 865.

[11] Morin, P., Perrier de la Bathie, R., à paraître. 